\title{
Investigation of Model Sunscreen Formulations Comparing the Sun Protection Factor, the Universal Sun Protection Factor and the Radical Formation Ratio
}

\author{
Felicia Syring Hans-Jürgen Weigmann Sabine Schanzer Martina C. Meinke \\ Fanny Knorr Jürgen Lademann \\ Center of Experimental and Applied Cutaneous Physiology, Department of Dermatology, Venerology and \\ Allergology, Charité - Universitätsmedizin Berlin, Berlin, Germany
}

\section{Key Words}

Sunscreen efficacy · Chemical filter · Physical filter .

Antioxidants · Human skin · Porcine ear skin · Electron

paramagnetic resonance spectroscopy

\begin{abstract}
In view of globally rising skin cancer rates and harmful effects exerted by sunlight throughout the ultraviolet, visible and infrared ranges, an objective, safe and comprehensive method for determining sunscreen efficacy is required in order to warrant safe sun exposure. In this study, the influence of characteristic active ingredients (chemical filters, physical filters and antioxidants) on different sunscreen indicators, including the universal sun protection factor and the radical formation ratio, was determined and compared to their influence on sun protection factor values. Spectroscopic universal sun protection factor measurements were conducted ex vivo by analyzing tape strips taken from human skin, and radical formation ratio determination was performed via electron paramagnetic resonance spectroscopy using porcine ear skin ex vivo. The sun protection factor determination was conducted according to ISO standards (ISO 24444: 2010). It was shown that chemical filters provide a protective effect which was measurable by all methods examined
\end{abstract}

\section{KARGER}

E-Mail karger@karger.com

www.karger.com/spp (spectroscopy, electron paramagnetic resonance spectroscopy and erythema formation). Physical filters, when used as single active ingredients, increased protective values in universal sun protection factor and sun protection factor measurements but exhibited no significant effect on universal sun protection factor measurements when used in combination with chemical filters or antioxidants. Antioxidants were shown to increase sun protection factor values. Radical formation ratio values were shown to be influenced merely by chemical filters, leading to the conclusion that the universal sun protection factor is the most suitable efficacy indicator for the ultraviolet range.

(c) 2015 S. Karger AG, Basel

\section{Introduction}

Changes in leisure activities and increasing skin cancer rates worldwide $[1,2]$ have led to a rising awareness regarding the importance of sun protection. While the acceptance of sunscreen use is surging, an objective, safe and comprehensive method for efficacy evaluation has become increasingly important.

Currently, the sun protection factor (SPF) is the accepted international standard for sunscreen efficacy eval- 
uation [1-4]. Based on erythema formation, it is a measure of how long sun exposure can be prolonged before sunburn is induced. However, by employing erythema formation as a single efficacy indicator, one not only neglects the consequences of radiation in volunteer testing but also focuses on a single biological process, which is predominantly induced by ultraviolet $B$ (UVB) radiation $[1,2,5]$. Damage known to be induced by ultraviolet $A$ (UVA) and even infrared (IR) and visible (VIS) radiation [6-8] can only be insufficiently accounted for by applying the SPF. Hence, the development of new efficacy indicators is of utmost importance.

Based on different mechanisms, several authors have proposed new methods to determine sunscreen efficacy in a more comprehensive manner [9-11], inter alia, the universal SPF (USPF) [12] and the radical formation ratio (RF) [13], which is based on electron paramagnetic resonance (EPR) measurements.

The USPF permits the objective quantification of the protective efficacy of sunscreen products throughout the entire UV range. This method is based on the sum transmission spectrum that is determined spectroscopically by employing the noninvasive tape stripping procedure in vivo. The resulting sum transmission quantifies the reduction in the UV radiation intensity achieved by a product and forms the basis for the calculation of a new protection factor.

A different approach is offered by the RF determined via EPR spectroscopy. Based on the findings of Zastrow et al. [8] that free radicals are not only produced in the UV range but also up to 50\% in the VIS and IR ranges, this method utilizes the underlying biological response of $\mathrm{RF}$ to determine the efficacy of sun protection, thus providing a possibility to determine the protective efficacy in the UV, VIS and IR ranges.

The aim of this study was to determine the influence of characteristic active ingredients on the USPF and RF and to compare them to the current sunscreen efficacy characterization standard, the SPF. While studies have shown reliable results for the EPR method in the IR and VIS ranges [14, 15], this study concentrated exclusively on the protection evaluation of these indicators in the UV range for comparative reasons.

For this purpose, commonly utilized active ingredients such as chemical filters (UVB and UVA filters), physical filters and antioxidant additives were added in defined amounts to different formulations and tested in a standardized setting.

The results provided an insight into the influence of different active ingredients on each efficacy indicator tested, demonstrating the relation between them. Ultimately, the results helped to determine the most suitable efficacy indicator in the UV range as part of a safe, objective and comprehensive sunscreen efficacy evaluation.

\section{Materials and Methods}

\section{Volunteers and Skin Samples}

For the spectroscopic measurements, 30 healthy volunteers (22 female and 8 male) aged 21 through 36 (mean 25.5 years) with skin type II or III on the Fitzpatrick skin type scale were selected. Measurements were conducted on untanned skin. No skin diseases were reported and no scars or visible damage to the skin were observed.

The study was conducted in agreement with the Declaration of Helsinki. Consent was given by each volunteer investigated and permission of the Ethics Committee of the Charité - Universitätsmedizin Berlin was obtained. EPR measurements were conducted on 6 fresh porcine ears provided by a local butcher. Ethical approval was obtained from the Veterinary Board Dahme-Spreewald, Germany.

\section{Sunscreen Formulations}

A total of 5 different formulations specifically prepared for the purpose of this study were used. For simplification purposes, the formulations were labeled with numbers 1, 2, 3, 4 and 5. The same base was used for each formulation (aqua, butylene glycol dicaprylate/dicaprate, glycerin, dioctylcyclohexane, polyglyceryl-2, dipolyhydroxystearate, glyceryl stearate, PEG-100 stearate, cetearyl alcohol, cetyl palmitate, magnesium aluminum silicate, xanthan gum, disodium ethylenediaminetetraacetic acid and preservative). The products only differed in their active ingredients: antioxidants $(1 \% \mathrm{w} / \mathrm{w}$; here bis-ethylhexyl hydroxydimethoxy benzylmalonate), chemical UV filters ( $12 \% \mathrm{w} / \mathrm{w}$; a combination of butyl methoxydibenzoylmethane and octocrylene) and physical filters ( $2 \%$ $\mathrm{w} / \mathrm{w}$ ) consisting of titanium dioxide. The active ingredients of each cream are summarized in table 1 .

The stability of the formulation was tested for 3 months at $40^{\circ} \mathrm{C}$ and with 10 cycles of freeze-thaw cycle, in which the formulation was frozen and thawed in a temperature interval of -5 to $40^{\circ} \mathrm{C}$. Phenoxyethanol and ethylhexylglycerin were used as preservatives in the formulations.

\section{Determination of the USPF}

In line with ISO standards (ISO 24444:2010), $160 \mathrm{mg}$ of sunscreen formulation was evenly distributed on a preferably hairless $8 \times 10 \mathrm{~cm}$ skin area on the inner forearm of the subjects. After 60 min of penetration time, to obtain a stable and almost homogeneous distribution of sunscreen on the skin, a series of 10 tape strips (tes $a^{\circledR}$ No. 5529; Beiersdorf, Hamburg, Germany) were taken from the same area of treated and untreated skin and immediately measured against an empty tape in the 240- to 500-nm range of a PerkinElmer Lambda 650 S UV/VIS spectrometer in front of the integrated sphere that was used in the transmittance mode. To obtain the absorbance capacity for each formulation, the influence of corneocytes on the spectra was corrected by subtracting the spectrum of the tape taken from untreated skin from the tape taken from treated skin using the UV WinLab program (UV WinLab 
Table 1. Summary of the formulations and their active ingredients

\begin{tabular}{ll}
\hline Formulation & Active ingredients \\
\hline Base & - \\
\hline Cream 1 & Antioxidants (1\%; bis-ethylhexyl hydroxydimethoxy benzylmalonate) \\
\hline Cream 2 & Physical UV filters (2\%; titanium dioxide) \\
\hline Cream 3 & Chemical UV filters (12\%; butyl methoxydibenzoylmethane, UVA filter and octocrylene, \\
& UVB filter) \\
\hline Cream 4 & Chemical UV filters and antioxidants \\
\hline Cream 5 & Chemical UV filters, physical UV filters and antioxidants \\
\hline
\end{tabular}

version 6.0.3.0730; PerkinElmer GmbH, Rodgau, Germany) and the UV WinLab Data Processor and Viewer (PerkinElmer 2009 version 1.00.00.0010).

The resulting spectra were summated in an absorbance scale and later changed to a transmission scale to determine the sum transmission spectrum. The area under this spectrum quantifies the transmitted light in the complete UV range (280-400 nm) after the application of a formulation. The average UV sum transmission - related to $1 \mathrm{~nm}$ - was obtained by dividing the value of the area under the curve by 120 , corresponding to the length of the complete UV range. This average sum transmission, which is given in percent, provided the basis for USPF calculations.

$\mathrm{USPF}=100$ /average UV sum transmission in percent

The resulting USPF values indicated, similarly to the SPF, to what degree sun exposure may be extended before sun-induced injuries occur, with the distinction that USPF values account for radiation of both the UVB and UVA ranges. The applicability and the relevance of the USPF value were demonstrated by investigating a large number of commercial sunscreens from the lowest up to high SPF values [16].

\section{Determination of the $R F$}

Porcine skin samples were cleaned, shaved and dried using a paper towel before a punch biopsy (19 $\mathrm{mm}$ in diameter) was removed from an unscathed part of the ears. A filter disc was then placed on the biopsy and treated with $50 \mu \mathrm{l}$ water:ethanol $(1: 1)$ $0.2 \%$ PCA solution (3-carboxy-2,2,5,5-tetramethylpyrrolidine1-oxyl; Sigma-Aldrich, Steinheim, Germany).

To avoid light-induced radical production, an occlusive and opaque covering was placed on the biopsy. The penetration time was set for $20 \mathrm{~min}$. Subsequently, $5.7 \mathrm{mg}\left(2 \mathrm{mg} / \mathrm{cm}^{2}\right.$; ISO 24444: 2010) of sunscreen formulation listed in table 1 was distributed homogenously on the sample. Following a 30-min induction period in an opaque chamber, the sample was placed in an L-band electron spin spectrometer (LBM MT 03; Magnettech, Berlin, Germany) and measured continuously for $16 \mathrm{~min}$. Successively, the sample was exposed to UV/VIS radiation and measurements were recorded for another $16 \mathrm{~min}$.

A solar simulator (LS0104; LOT, Darmstadt, Germany) containing a 150-watt Xenon arc lamp was utilized for this study. The corresponding settings have been described in detail by Meinke et al. [14]. The irradiation intensity was measured at $90 \mathrm{~mW} / \mathrm{cm}^{2}$. UVA was $8.55 \mathrm{~mW} / \mathrm{cm}^{2}$, UVB $1.75 \mathrm{~mW} / \mathrm{cm}^{2}$ and VIS $79.9 \mathrm{~mW} /$ $\mathrm{cm}^{2}$ (ILT 1400 Radiometer Photometer; Polytec, Waldbronn, Germany). After $16 \mathrm{~min}$, the accumulated energy yielded $86.4 \mathrm{~J} / \mathrm{cm}^{2}$.

MPlot.exe was used for the EPR signal determination. Peak-topeak measurements were conducted in the central line of the spectrum and measurements were normalized to the initial value to account for peak intensity variations between skin samples. The $\mathrm{RF}$ was then determined by establishing a ratio of mean signal intensity before and after UV/VIS irradiation.

$\mathrm{RF}=\mathrm{EPR}$ signal before irradiation/EPR signal after irradiation

\section{Determination of the SPF}

The in vivo SPF was determined for 4 formulations (creams 2, 3,4 and 5) by proDERM GmbH according to ISO standards (ISO 24444:2010). To determine the SPF values, each forearm of every volunteer was partly treated with the formulation and partly left untreated. After the penetration time, the volunteers underwent irradiation. Later on, both the treated and untreated areas were inspected for redness, and the minimal erythemal dose (MED), which is the lowest UV dose that produces redness, was determined. The SPF was then calculated by dividing the MED of the protected skin by the MED of the unprotected skin, determining how long sun exposure can be extended before burning occurs.

\section{Statistical Analysis}

Statistical analysis of the results was performed using IBM SPSS Statistics version 20 and Microsoft Excel for Mac 2011. A p value of $\leq 0.05$ was found to be statistically significant. A trend could be observed when $\mathrm{p} \leq 0.1$. The Kruskal-Wallis and Mann-Whitney tests were used to establish significant differences between the independent nonparametric mean values obtained.

\section{Results and Discussion}

By investigating the influence of the different models of creams on the selected efficacy indicator separately, the following results were obtained. Mean USPF values (fig. 1) were shown to be significantly increased by chemical filters present in the investigated formulations. The absorbance properties of the added filters led to a significant light attenuation. Furthermore, a small but statistically 


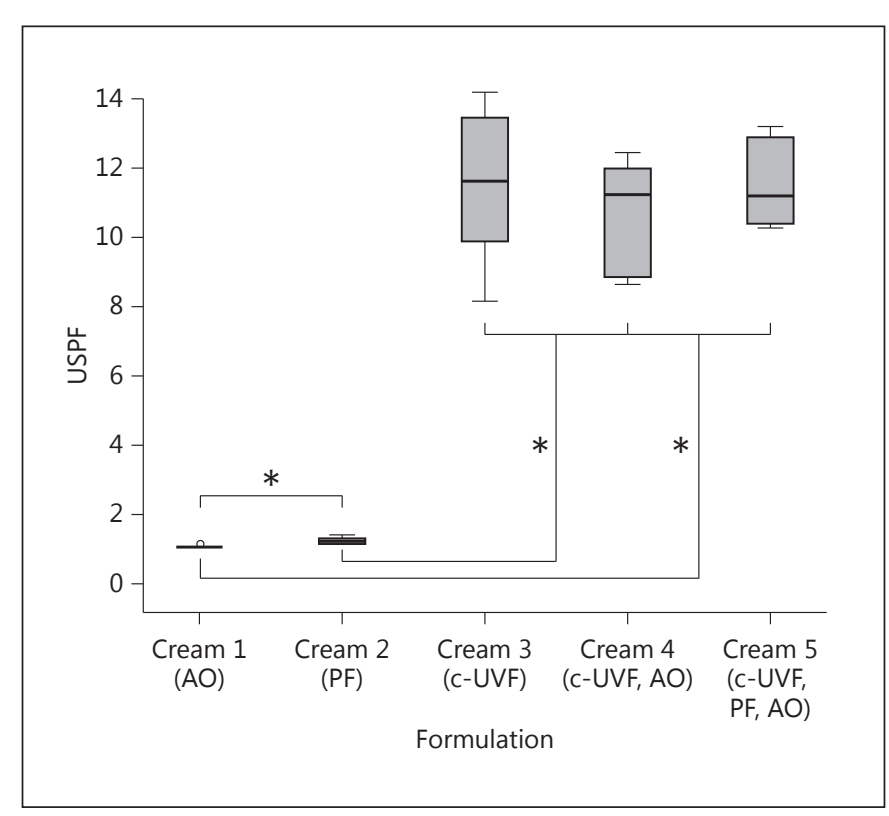

Fig. 1. USPF value box plot for each tested formulation. $A O=A n-$ tioxidants; $\mathrm{PF}=$ physical filter; $\mathrm{c}-\mathrm{UVF}=$ chemical filter. ${ }^{*} \mathrm{p}<0.05$, significant difference between creams ( $\mathrm{n}=6$ for each cream).

significant difference between cream 1 , containing antioxidants, and cream 2 , made with physical filters, was noted (1.05 and 1.24, respectively). On the one hand, this reflects the anticipated lack of effect of antioxidants as they did not influence the intensity of incident radiation, and on the other hand, it illustrates a small yet expected light attenuation due to the reflection and absorption properties of the physical filter. However, the observed protective efficacy of the physical filters could not be reproduced in measurements for creams also containing chemical filters. The lack of effect is attributable to the relatively small amount $(2 \%)$ of physical filters added, resulting in a low extinction which does not remarkably influence the high values of the creams containing chemical filters. This can be explained by the much larger individual variation between USPF values for creams containing chemical filters, concealing smaller variations in light attenuation. Previous research has shown that increasing the doses of physical filters increases the protective efficacy of formulations [17], which is reflected in the USPF mean values. These results reinforce the use of the spectroscopic USPF as an objective measure of protective efficacy that is independent of biological processes and covers both the UVB and UVA ranges.

Figure 2 illustrates the RF after 16 min. EPR measurements show a significant difference between creams con-

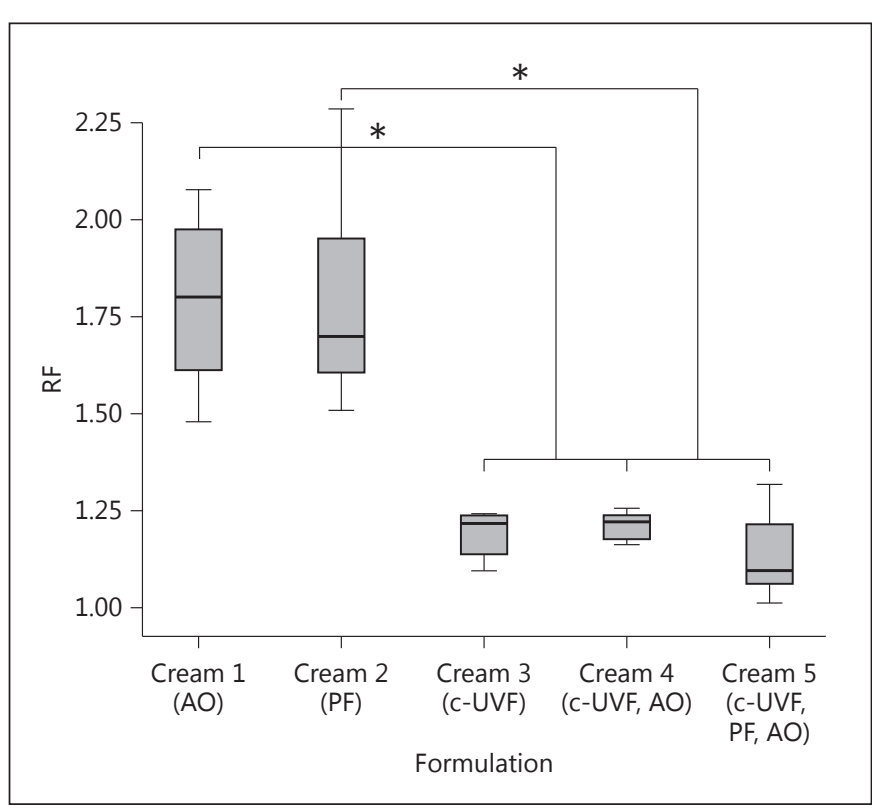

Fig. 2. The RF after 16 min for each formulation. $A O=$ Antioxidants; $\mathrm{PF}=$ physical filter; $\mathrm{c}-\mathrm{UVF}=$ chemical filter. ${ }^{*} \mathrm{p}<0.05$, significant difference between creams $(\mathrm{n}=6$ for creams $1-4, \mathrm{n}=7$ for cream 5).

taining no chemical filters (creams 1 and 2) and creams containing absorbing chemical filters (creams 3, 4 and 5). No significant differences between either creams 1 and 2 or creams 3, 4 and 5 were observed. This agrees with the small changes observed for the spectroscopic values of the corresponding creams. Contrary to expectations, antioxidants had no measurable statistically significant effect in the UV region. While previous research has shown the radical scavenging potential of topically applied antioxidants $[18,19]$ and has affirmed EPR spectroscopy as a suitable method for detection, this was not reproducible in this study. A possible explanation may be the relatively low amount of antioxidants added (1\%) as well as the high ex vivo radiation intensity used in this study. In order to enhance the sensitivity of the measurements, a 20 -fold MED was utilized in this setting, thereby possibly obliterating antioxidants early on.

Additionally, no effect of the physical filter was observed, which was not predicted. Previously, Meinke et al. [20] showed that not only antioxidants but also high scattering properties led to a significant reduction of radical formation in the near IR region. Physical filters primarily function by means of scattering and reflection, thereby preventing radiation from penetrating into deeper skin layers, and while free radicals may be produced on a smaller scale in the upper strata, excess free radical formation primarily 


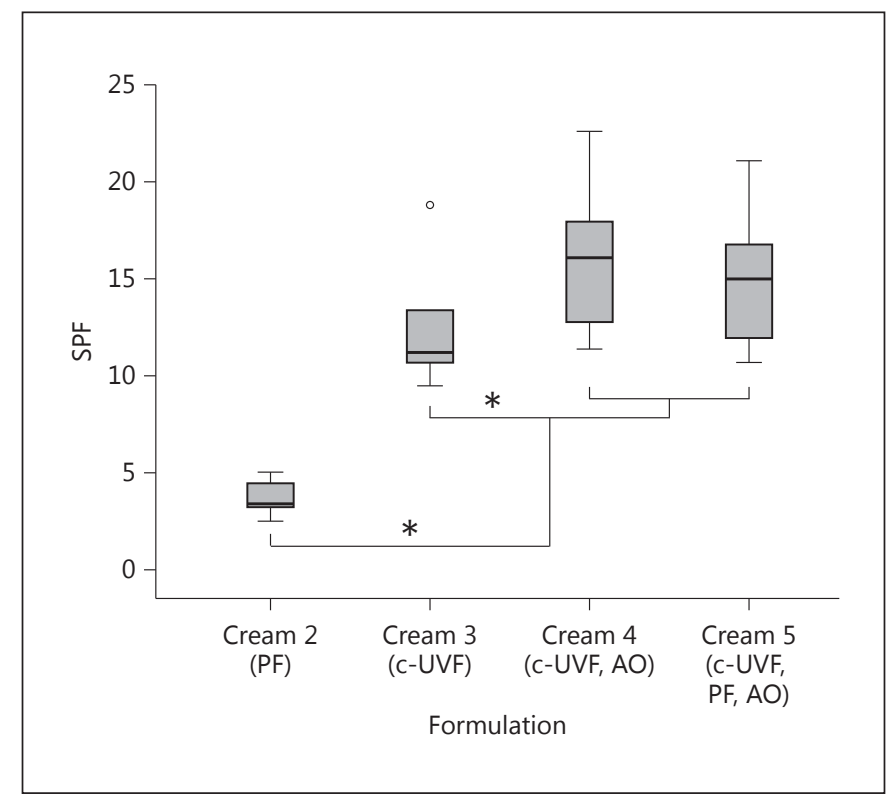

Fig. 3. SPF value box plot for each tested formulation. $\mathrm{AO}=$ Antioxidants; $\mathrm{PF}=$ physical filter; $\mathrm{c}-\mathrm{UVF}=$ chemical filter. ${ }^{*} \mathrm{p}<0.05$, significant difference between creams $(\mathrm{n}=10$ for each cream).

produced in the deeper skin layers should be averted. Similarly to the small effects noted in USPF measurements, an explanation for the lack of effect may be provided by the low amount of physical filters present in the formulations (2\%). Previous research employing a similar filter in a higher amount (5\%) showed a substantial decrease of radical formation in the UV region, suggesting too little active ingredient for a measurable effect in this setting.

SPF testing was conducted by proDERM GmbH according to ISO standards (ISO 24444:2010). The results are summarized in figure 3. Chemical filters were again shown to be of clear protective value. Additionally, statistical testing disclosed a significant difference between cream 3, containing chemical filters as single active ingredients, and creams 4 and 5, which contained, in addition to the chemical filters, antioxidants (cream 4) as well as antioxidants and physical filters (cream 5). This had not been observed during USPF and RF testing, despite it being evidenced in previous research. It could be demonstrated that topically applied antioxidants exhibit protective effects during UV irradiation [21,22], whereas previously, diets rich in antioxidants had been shown to reduce erythema formation [23]. As cream 1 was not tested, no conclusions regarding the protective effects of antioxidants independent of the presence of chemical filters could be drawn in this study. SPF testing for the physical filter alone (cream 2 ) resulted in a mean protective value of 3.7. This is on par with our expectations as we estimated an increase of 2 SPF units for every percent of physical filter added.

However, previously described synergistic effects between physical filters and organic filters [24] could not be seen. The only cream containing both chemical and physical filters in addition to antioxidants did not provide a significant increase in the mean SPF value during testing when compared to cream 4 , containing only chemical filters and antioxidants. No cream containing only physical and chemical filters was tested.

As Meinke et al. [14] pointed out, a possible explanation for this finding may lie within the antioxidant capacity of each cream. When the radical protection factor (which serves to express the antioxidative capacity in the creams) for these creams was determined, a much lower radical protection factor was seen for cream 5 [(29 \pm 1$)$ $10^{14}$ radicals $\left./ \mathrm{mg}\right]$ than for creams $1\left[(444 \pm 22) 10^{14} \mathrm{rad}-\right.$ icals $/ \mathrm{mg}]$ and 4 [(459 \pm 28$) 10^{14}$ radicals $\left./ \mathrm{mg}\right]$.

These results suggest that the antioxidant capacity may change when used in combination with physical filters. Hence, the SPF value seen in cream 5 is possibly a product of chemical and physical filters displaying the synergistic effects predicted rather than the antioxidants added in the cream. However, this hypothesis is contrary to the USPF values for creams 4 and 5, in which no synergistic effects of chemical and physical filters can be evidenced.

Lastly, efficacy indicators were analyzed for possible correlations. Although based on different mechanisms, due to the previously discussed lack of effect of antioxidants in the EPR measurements and little effect of physical filters in USPF mean values, the predominating protective effect was provided by chemical filters in both methods. From figures 1 and 2 it becomes obvious that high USPF values and low RF values were found for all creams containing chemical filters, suggesting a clear correlation between both efficacy indicators.

\section{Conclusion}

Chemical filters were shown to provide a protective effect in all three methods. Physical filters increased protection factors for USPF and SPF measurements as single active ingredients. However, they failed to show an effect when combined with chemical filters. Finally, antioxidants exhibited a protective effect during SPF testing.
Syring/Weigmann/Schanzer/Meinke/ Knorr/Lademann 
In conclusion, the USPF is the most suitable efficacy indicator for both UV ranges (UVA and UVB). It is independent of biological damages, is determined in a noninvasive setting, objectively quantifies the protective efficacy of a sunscreen and is impartial to additives in formulations. Although the RF correlates significantly with the USPF in the current setting, it is less suited to evaluate sunscreen efficacy in the UV range. In this setting, high irradiation intensity had to be used to increase the sensitivity of the measurements, which therefore led to an obliteration of antioxidants early on, consequently preventing clear conclusions to be drawn regarding the protective efficacy of the antioxidants measured. Furthermore, the RF is based on a biological process susceptible to a variety of factors leading to changes in radical formation, such as the addition of supplemental anti- inflammatory substances to a sunscreen, increased stress levels in the subject studied or differences in diet increasing or decreasing antioxidant capacity. Hence, it is less suited for an aspired objective evaluation in the UV spectrum.

\section{Acknowledgments}

We would like to thank Merck KGaA for providing the creams, as well as Dr. Pflücker and Manuela Loch for the fruitful discussions.

\section{Disclosure Statement}

The authors have no conflicts of interest to declare.

\section{References}

1 Godar DE: Worldwide increasing incidences of cutaneous malignant melanoma. J Skin Cancer 2011;2011:858425.

2 Rogers HW, Weinstock MA, Harris AR, Hinckley MR, Feldman SR, Fleischer AB, Coldiron BM: Incidence estimate of nonmelanoma skin cancer in the United States, 2006. Arch Dermatol 2010;146:283-287.

3 Kluschke F, Weigmann HJ, Schanzer S, Meinke M, Vergou T, Sterry W, Lademann J: Gain or loss? Sunscreen efficiency after cosmetic pretreatment of the skin. Skin Pharmacol Physiol 2014;27:82-89.

4 Vergou T, Patzelt A, Schanzer S, Meinke MC, Weigmann HJ, Thiede G, Sterry W, Lademann J, Darvin ME: Methods for the evaluation of the protective efficacy of sunscreen products. Skin Pharmacol Physiol 2013;26: 30-35.

5 Weigmann HJ, Schanzer S, Vergou T, Antoniou C, Sterry W, Lademann J: Quantification of the inhomogeneous distribution of topically applied substances by optical spectroscopy: definition of a factor of inhomogeneity. Skin Pharmacol Physiol 2012;25:118-123.

6 Schwarz A, Schwarz T: UVR-induced regulatory $\mathrm{T}$ cells switch antigen-presenting cells from a stimulatory to a regulatory phenotype. J Invest Dermatol 2010;130:1914-1921.

7 Waris G, Ahsan H: Reactive oxygen species: role in the development of cancer and various chronic conditions. J Carcinog 2006;5:14.

8 Zastrow L, Groth N, Klein F, Kockott D, Lademann J, Renneberg R, Ferrero L: The missing link - light-induced (280-1,600 nm) free radical formation in human skin. Skin Pharmacol Physiol 2009;22:31-44.

9 Zastrow L, Ferrero L, Herrling T, Groth N: Integrated sun protection factor: a new sun protection factor based on free radicals gener- ated by UV irradiation. Skin Pharmacol Physiol 2004;17:219-231.

10 Herrling T, Jung K, Chatelain E, Langenauer M: Radical skin/sun protection factor RSF protection against UV-induced free radicals in skin. SOFW J 2006;132:24-30.

11 Weigmann HJ, Schanzer S, Antoniou C, Herrling J, André V, Wünsch T, Schaefer H, Sterry W, Lademann J: Development of a universal sunscreen protection factor (USPF) based on ex vivo spectroscopic measurements. SOFW J 2007;133:2-7.

12 Weigmann H-J, Schanzer S, Teichmann A, Durat F, Antoniou C, Schaefer H, Sterry W, Lademann J: Ex-vivo spectroscopic quantification of sunscreen efficacy: proposal of a universal sun protection factor. J Biomed Opt 2007;12:044013.

13 Syring FM: The future of sunscreen efficacy evaluation. A comparative study of universal sun protection factor, radical formation ratio and sun protection factor assessing the protective value of sunscreen formulations containing chemical-, and/or physical filters as well as antioxidant additives; thesis, Charité, Berlin, 2015.

14 Meinke MC, Syring F, Schanzer S, Haag SF, Graf R, Loch M, Gersonde I, Groth N, Pflucker F, Lademann J: Radical protection by differently composed creams in the UV/VIS and IR spectral ranges. Photochem Photobiol 2013;89:1079-1084.

15 Lauer AC, Groth N, Haag SF, Darvin ME, Lademann J, Meinke MC: Dose-dependent vitamin $\mathrm{C}$ uptake and radical scavenging activity in human skin measured with in vivo electron paramagnetic resonance spectroscopy. Skin Pharmacol Physiol 2013;26:147-154. 16 Weigmann HJ, Schanzer S, Antoniou C, Sterry W, Lademann J: Influence of the absorp- tion behavior of sunscreens in the shortwavelength UV range (UVB) and the longwavelength UV range (UVA) on the relation of the UVB absorption to sun protection factor. J Biomed Opt 2010;15:055008.

17 Mitchnick MA, Fairhurst D, Pinnell SR: Microfine zinc oxide (Z-cote) as a photostable UVA/UVB sunblock agent. J Am Acad Dermatol 1999;40:85-90.

18 Darr D, Combs S, Dunston S, Manning T, Pinnell S: Topical vitamin C protects porcine skin from ultraviolet radiation-induced damage. Br J Dermatol 1992;127:247-253.

19 Jurkiewicz BA, Bissett DL, Buettner GR: Effect of topically applied tocopherol on ultraviolet radiation-mediated free radical damage in skin. J Invest Dermatol 1995;104:484-488.

20 Meinke MC, Haag SF, Schanzer S, Groth N, Gersonde I, Lademann J: Radical protection by sunscreens in the infrared spectral range. Photochem Photobiol 2011;87:452-456.

21 Dreher F, Denig N, Gabard B, Schwindt DA, Maibach HI: Effect of topical antioxidants on UV-induced erythema formation when administered after exposure. Dermatology 1999;198:52-55.

22 Lin JY, Selim MA, Shea CR, Grichnik JM, Omar MM, Monteiro-Riviere NA, Pinnell SR: UV photoprotection by combination topical antioxidants vitamin $\mathrm{C}$ and vitamin E. J Am Acad Dermatol 2003;48:866-874.

23 Stahl W, Heinrich U, Aust O, Tronnier H, Sies $\mathrm{H}$ : Lycopene-rich products and dietary photoprotection. Photochem Photobiol Sci 2006; 5:238-242.

24 Lademann J, Schanzer S, Jacobi U, Schaefer H, Pflucker F, Driller H, Beck J, Meinke M, Roggan A, Sterry W: Synergy effects between organic and inorganic UV filters in sunscreens. J Biomed Opt 2005;10:14008.
Sunscreen Efficacy: A Comparison of Characterization Approaches
Skin Pharmacol Physiol 2016;29:18-23 DOI: $10.1159 / 000441037$ 